OPEN

SUBJECT AREAS:

MITOSIS

BIOMIMETICS

EMBRYONIC STEM CELLS

Received

6 August 2013

Accepted

25 November 2013

Published

11 December 2013

Correspondence and requests for materials should be addressed to T.N.S. (island1005@ bs.naist.jp)

\section{Synthetic cell division system: Controlling equal vs. unequal divisions by design}

\author{
Yoichi Sato', Kazuma Yasuhara', Jun-ichi Kikuchi \& Thomas N. Sato ${ }^{1,3,4,5,6}$
}

${ }^{1}$ Graduate School of Biological Sciences, Nara Institute of Science and Technology, Nara, Japan, ${ }^{2}$ Graduate School of Materials Science, Nara Institute of Science and Technology, Nara, Japan, ${ }^{3}$ Department of Biomedical Engineering, Cornell University, Ithaca, NY, USA, ${ }^{4}$ Centenary Institute, Sydney, Australia, ${ }^{5}$ ERATO Sato Live Bio-Forecasting Project, Japan Science and Technology Agency, Kyoto, Japan, ${ }^{6}$ Advanced Telecommunications Research Institute International (ATR), Kyoto, Japan.

Cell division is one of the most fundamental and evolutionarily conserved biological processes. Here, we report a synthetic system where we can control by design equal vs. unequal divisions. We synthesized a micro-scale inverse amphipathic droplet of which division is triggered by the increase of surface to volume ratio. Using this system, we succeeded in selectively inducing equal vs. unequal divisions of the droplet cells by adjusting the temperature or the viscosity of the solvent outside the droplet cell accordingly. Our synthetic division system may provide a platform for further development to a system where intracellular contents of the parent droplet cell could be divided into various ratios between the two daughter droplet cells to control their functions and fates.

r he goal of synthetic biology is to design and synthesize artificial system that mimics or that is distinct from and more efficient than the naturally occurring biological processes. Cell division is a central biological process that is essential for any forms of life for their reproduction and propagation, and is conserved through evolution.

Stem cells make decisions on either self-reproduction or differentiation by selectively utilizing equal vs. unequal cell divisions ${ }^{1,2}$. Stem cells reproduce themselves by equal divisions. However, they undergo a one-round of an unequal division to reproduce one daughter stem cell which is identical to the original and to produce the other daughter cell that is destined to differentiate to a specialized cell type, thus differentiating into non-stem cell lineages - the process known as "asymmetric cell division". The differentiated cells reproduce themselves via equal divisions. Therefore, the ability to control equal vs. unequal divisions by design provides a unique synthetic system capable of making decisions on its future fates and states by selectively utilizing two types of divisions.

Over the last two decades, several synthetic cell division systems were synthesized ${ }^{3-13}$. However, none provided a system where equal vs. unequal divisions could be controlled by design. Hence, we set out to synthesize an artificial cell system where we can control equal vs. unequal divisions by design.

\section{Results}

First, we synthesized an artificial cell division system based on a simple physical law. The assumption that we made is that a synthetic cell would divide if the ratio of the surface area to volume increases. To accomplish this goal, we developed a division system of inverse amphipathic droplet cell where the surface area could be increased by hydrolysis reaction (Fig. 1a, b). The droplet was formed by a mixture of sorbitan monooleate (Span 80) and polyoxyethylene sorbitan monooleate (Tween 80 ) with alkaline phosphate buffer solution inside and hydrophobic oil (mineral oil or liquid paraffin) outside (i.e. inverse amphipathic system) (Fig. 1a) (see also Materials and Methods for the details). p-nitrophenyl palmitate (pNPP), a previously used substrate for lipase activity assay within lipid micelles ${ }^{14}$, was added to the outside oil phase, and as pNPP molecules contact the inside alkaline phosphate phase in a spontaneous manner, pNPP is hydrolyzed and the palmitate molecules are incorporated to the hydrophobic droplet surface (Fig. 1a, b). As more pNPP molecules are hydrolyzed and the number of palmitate molecules within the hydrophobic phase surface increases, the droplet surface area expands. When the surface area reaches to the critical threshold while the total droplet volume remains constant (i.e. the increase of the surface area to volume ratio), the droplet is expected to divide (Fig. 1a, b).

The inverse amphipathic droplets divided following the hydrolysis reaction. Furthermore, we were able to induce distinct types of divisions depending on the temperatures or the viscosities of solvents outside the droplets (Fig. 2). At $30^{\circ} \mathrm{C}$ in mineral oil solvent, the single parent droplet divided into two daughter droplets of nearly equal 
a

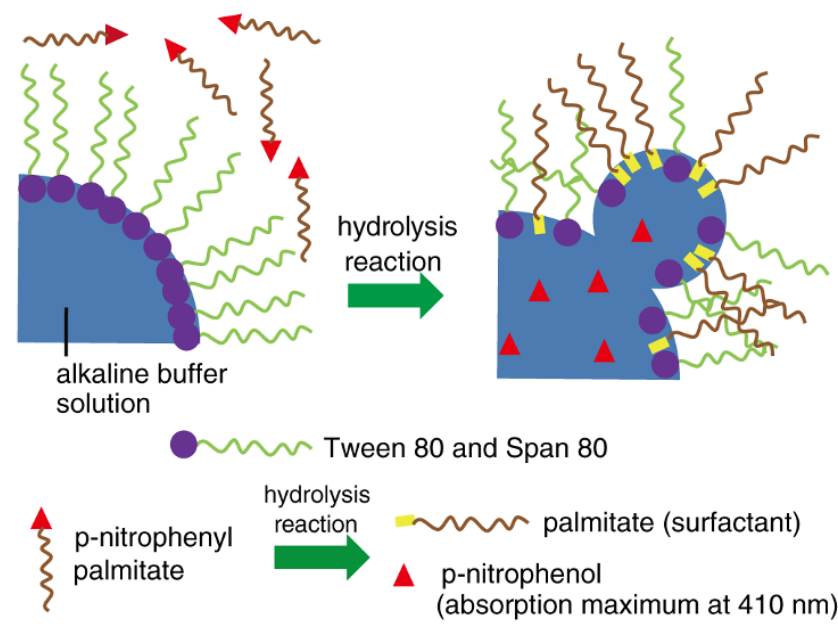

b

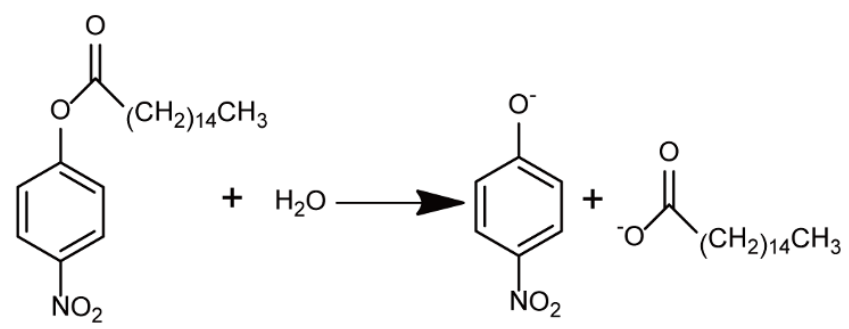

Figure $1 \mid$ Schematic representation of a synthetic cell division system. (a). Outline of the division system. Incorporation of palmitate produced from pNPP by hydrolysis increases the surface to volume ratio of the droplet cell, which triggers the division. (b). Hydrolysis of hydrophobic pNPP producing amphipathic palmitate.

size, i.e., the equal cell division (Fig. 2a and Supplementary Video 1). In contrast, at $25^{\circ} \mathrm{C}$ in mineral oil solvent, a single parent droplet divided into two daughters of smaller and larger sizes, i.e. an unequal cell division (Fig. 2b and Supplementary Video 2). In liquid paraffin, that exhibits much lower viscosity than mineral oil (Supplementary Table S1), the single parent droplet divided into multiple smaller daughter droplets, i.e., multiple buddings, at $25^{\circ} \mathrm{C}$ (Fig. $2 \mathrm{c}$ and Supplementary Video 3). We observed some volume loss of the daughter cells following the divisions (Fig. 2a, 2b, Supplementary Videos 1,2). This is presumably due to the fact that the daughter droplets rapidly float away from the observable field under the microscope following the division. This is unavoidable because each droplet is floating in the liquid phase during the microscopy imaging. Alternatively, there may be some leakage of solvent inside the droplets following the divisions. Next, we also examined the divisions in solvents of intermediate viscosities and investigated their effects on the sizes of the daughter droplet cells (Fig. 2d). By mixing mineral oil and liquid paraffin at various ratios, we generated the solvents of which viscosities falls in between those of mineral oil and liquid paraffin (Supplementary Table S1), and we examined the droplet cell divisions in each of the solvent of the intermediate viscosities at $25^{\circ} \mathrm{C}$ (Fig. $2 \mathrm{~d}$ ). The result shows that the sizes of two daughter cells became closer to equal (i.e., semi-equal division) as the solvent viscosity decreases (Fig. 2d). Taken all these results together, we conclude that the droplet cell undergoes an equal division in a solvent of high viscosity (i.e., mineral oil) at $30^{\circ} \mathrm{C}$ (Fig. 2e). However, the droplet cell undergoes unequal division, producing two daughter cells of distinct sizes, in the same solvent (i.e., mineral oil) but at lower temperature, i.e., $25^{\circ} \mathrm{C}$ (Fig. 2e). The size difference of two daughter cells produced by the unequal divisions become less prominent as the droplet cells divide in the solvents of lower viscosities at $25^{\circ} \mathrm{C}$ (Fig. 2e). A solvent of low viscosity (i.e., liquid paraffin) at $25^{\circ} \mathrm{C}$ induces multiple buddings (Fig. 2e).

Next we examined the mechanism underlying the division of the synthetic droplet cell. The hydrolysis reaction was $\mathrm{pH}$ dependent i.e., the reaction is more efficient at the higher $\mathrm{pH}$. The divisions of droplets were monitored at $\mathrm{pH} 8$ - $\mathrm{pH} 13$ in mineral oil and liquid paraffin (Fig. 3a). In mineral oil and liquid paraffin, the transitions to the division occurred at $\mathrm{pH} 11$ and $\mathrm{pH} 10$, respectively, demonstrating the $\mathrm{pH}$ dependency of the divisions. In addition, the droplet division occurred at a narrow range of critical concentration of palmitate, the product of the hydrolysis of pNPP (Fig. 3b). Over $70 \%$ of the droplets divided at $5.4 \times 10^{-5} \mathrm{M}$ and $2.4 \times 10^{-5} \mathrm{M}$ in mineral oil and liquid paraffin, respectively (Fig. 3b). The required concentration of palmitate for the division to occur appears to increase as the viscosity of the solvent increases (Fig. 3c). Quantitative measurements of both the surface area and the volume of individual droplets confirmed that the division occurred as the surface area increased while the volume remained constant in both mineral oil (Fig. 3d) and liquid paraffin (Fig. 3e). These results are all in agreement with the idea that the droplet division is dependent on hydrolysis of hydrophobic molecule, pNPP, to amphipathic molecule, palmitate, and the division results from the concentration increase of palmitate which becomes incorporated into the amphipathic droplet surface, thus increasing the surface to volume ratio. The results also demonstrate that more palmitate molecules are required for the divisions to occur in the solvents with higher viscosities, as indicated by the higher critical concentration threshold for the division in the higher viscosity solvents.

\section{Discussion}

Herein we report an inverse amphipathic droplet division system where the division can be induced by supplying pNPP to the outside of the lipid droplets, and furthermore, the equal vs. unequal divisions can be controlled by adjusting temperatures and viscosities of the solvents. Although several other self-replicating synthetic cell division systems have been previously reported, to our knowledge, this is the first system where the equal vs. unequal divisions can be selectively induced depending on the temperatures and viscosities of the microenvironment.

Our results are consistent with a model where pNPP is hydrolyzed in alkaline microenvironment producing palmitate which becomes incorporated into the droplet surface, thus increases the droplet surface to volume ratio and results in the division (Figs. 1, 3). Another possible mechanism for consideration is a contribution of surface energy effects to the initiation of the observed droplet division, a possibility that remains for a future investigation.

The system reported here is an inverse system (i.e. the inside of the droplet is hydrophilic), thus it is possible to enclose biomolecules such as proteins and nucleic acids that are relatively hydrophilic. Such macromolecules and other agents could be inherited to daughter droplets at various ratios by incorporating them inside the droplets and inducing the divisions producing two daughter cells of various size ratios. Hence, the system could be further developed to exert functional variations between two daughter cells through unequal divisions.

Most natural cells existing in modern days use cytoskeleton based force generator system for the division ${ }^{15}$, however, it has been recently discovered that some modern bacteria retain the ability to switch to a state referred to as L-form of which division is induced by increasing cell surface to noise ratio ${ }^{16}$. The study also suggested that the increasing the surface to volume ratio represents the most primitive strategy of division that primordial cells exploited in early days in evolution ${ }^{16}$. Our results reported herein suggest that a lipid droplet division driven by an increased surface to volume is possible. 
a

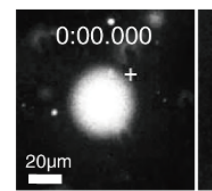

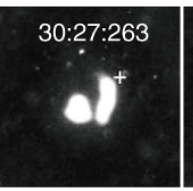

C

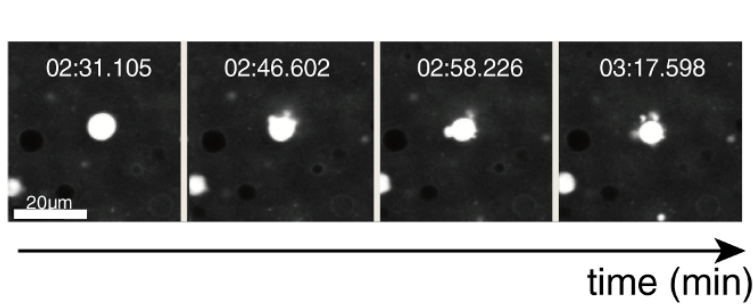

b

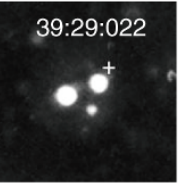

time $(\mathrm{min})$

d
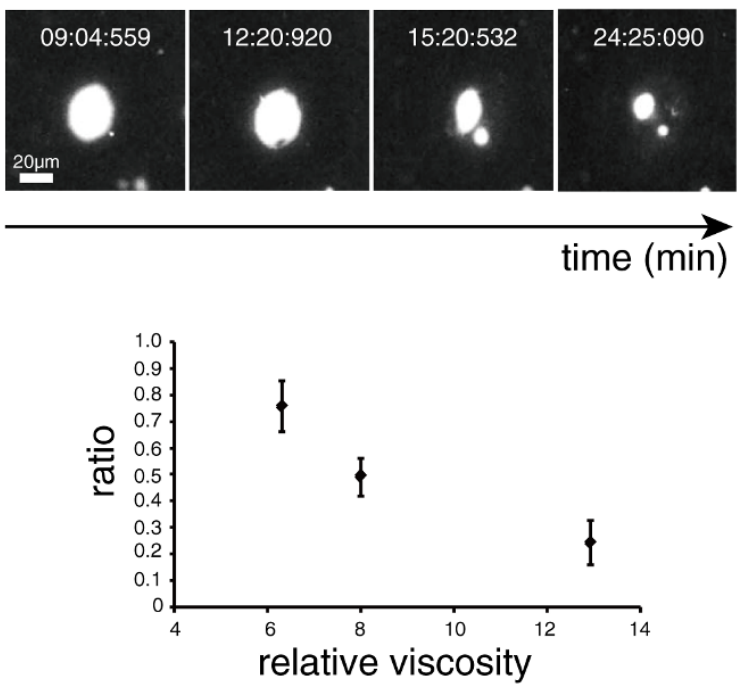

e

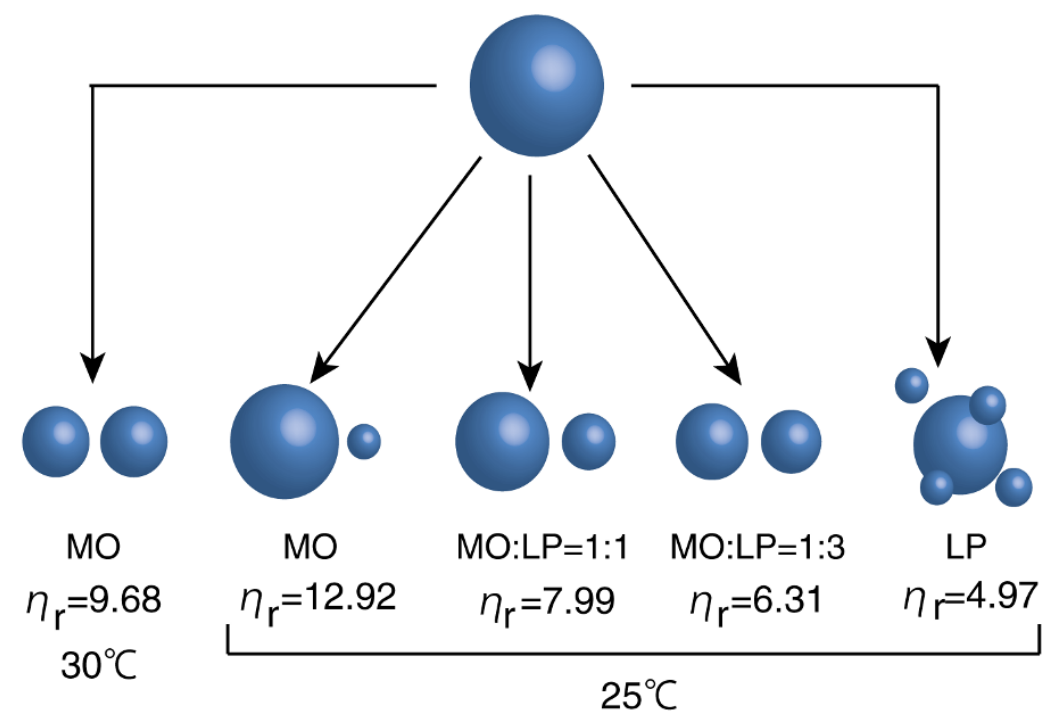

Figure $2 \mid$ Distinct division types induced by different temperatures and solvent viscosities. (a). Division in mineral oil at $30^{\circ} \mathrm{C}$. See also Supplementary video $1 . \mathrm{n}=30$, Ratio $=0.94 \pm 0.05$ (of two daughter droplet cell diameters). (b). Division in mineral oil at $25^{\circ} \mathrm{C}$. See also Supplementary video $2 . \mathrm{n}=31$, Ratio $=0.41 \pm 0.09$ (of two daughter droplet cell diameters). (c). Division in liquid paraffin at $25^{\circ} \mathrm{C}$. See also Supplementary video $3 . \mathrm{n}=31$.

Scale bars (a-c): $20 \mu \mathrm{m}$. Scale bars (Supplementary videos): $50 \mu \mathrm{m}$. Time stamps (Supplementary videos): day (d):hours:minutes:seconds:milliseconds. (d). Ratios of two daughter cell sizes (in diameters) after the division in solvents of intermediate viscosities at $25^{\circ} \mathrm{C} . \mathrm{n}=30\left(\right.$ for $\left.\eta_{\mathrm{r}}=6.31\right), \mathrm{n}=30\left(\eta_{\mathrm{r}}=\right.$ 7.99), $\mathrm{n}=31$ (for $\eta_{\mathrm{r}}=12.92$ ). One-way ANOVA test; $\mathrm{p}<5 \times 10^{-7}$. (e). Schematic diagram representing various division types. $\eta_{\mathrm{r}}$ : relative viscosity. MO: mineral oil, LP: liquid paraffin.

Furthermore, our studies clearly show that temperature and viscosity of the microenvironment influence the division types (e.g., equal vs. unequal divisions, and multiple buddings). Hence, an intriguing possibility, but is subject to rigorous testing in the future, is that an environment of dynamically changing temperature and viscosity may have contributed to the evolution of different types of naturally existing cell divisions (e.g., equal and unequal divisions, multiple buddings).

\section{Methods}

Preparation of amphipathic droplets. Amphipathic droplets were synthesized as follows: Sorbitan monooleate (Span 80) (Wako Pure Chemical Industry Co., Japan) $(0.21 \mathrm{~g})$ (final conc.: $0.49 \mathrm{mM}$ ), polyoxyethylene sorbitan monooleate (Tween 80 ) $(0.01 \mathrm{~g})$ (final conc.: $76 \mu \mathrm{M})$, Na-phosphate buffer $(1 \mathrm{~g})$ and p-nitrophenyl palmitate (pNPP) (MP Biochemicals Inc., USA) $(0.35 \mathrm{~g})$ (final conc.: $1 \mathrm{mM}$ ) were sequentially added to either mineral oil Light White (MP Biochemicals Inc., USA) or to liquid paraffin (Wako Pure Chemical Industry Co., Japan) (8.78 g for both oils). The amphipathic droplets were prepared by vigorously mixing each sample by vortexing for $3 \mathrm{~min}$. at either $25^{\circ} \mathrm{C}$ or $30^{\circ} \mathrm{C}$.

Confocal microscopy. The droplet divisions were observed in the $35 \mathrm{~mm}$ glassbottom dish (IWAKI \& CO., Ltd.) maintained at either $25^{\circ} \mathrm{C}$ or $30^{\circ} \mathrm{C}$ using LSM$7 D U O$ confocal laser microscopy (Carl Zeiss, Inc.) with line mode and $40 \times$ wateremulsion lens (NA1.2). Each droplet was visualized by the inclusion of Rhodamine B (Wako Pure Chemical Industry Co., Japan) in the Na-phosphate buffer (final conc.: $0.35 \mathrm{mM}$ ) and the time-lapse images were archived.

Measurement of palmitate concentration. The concentration of palmitate in the alkaline aqueous phase was calculated by measuring the absorbance of p-nitrophenol, the by-product of the hydrolysis reaction ( $\mathrm{pNPP} \rightarrow$ palmitate $+\mathrm{p}$-nitrophenol), at $410 \mathrm{~nm}$ using Shimadzu UV-2400PC (Fig. 1a). The amphipathic droplets emulsion was diluted with acetone prior to measuring the absorbance.

Measurements of droplet size. The diameter of individual amphipathic droplets was measured based on the confocal microscopy images using ImageJ and was used to 


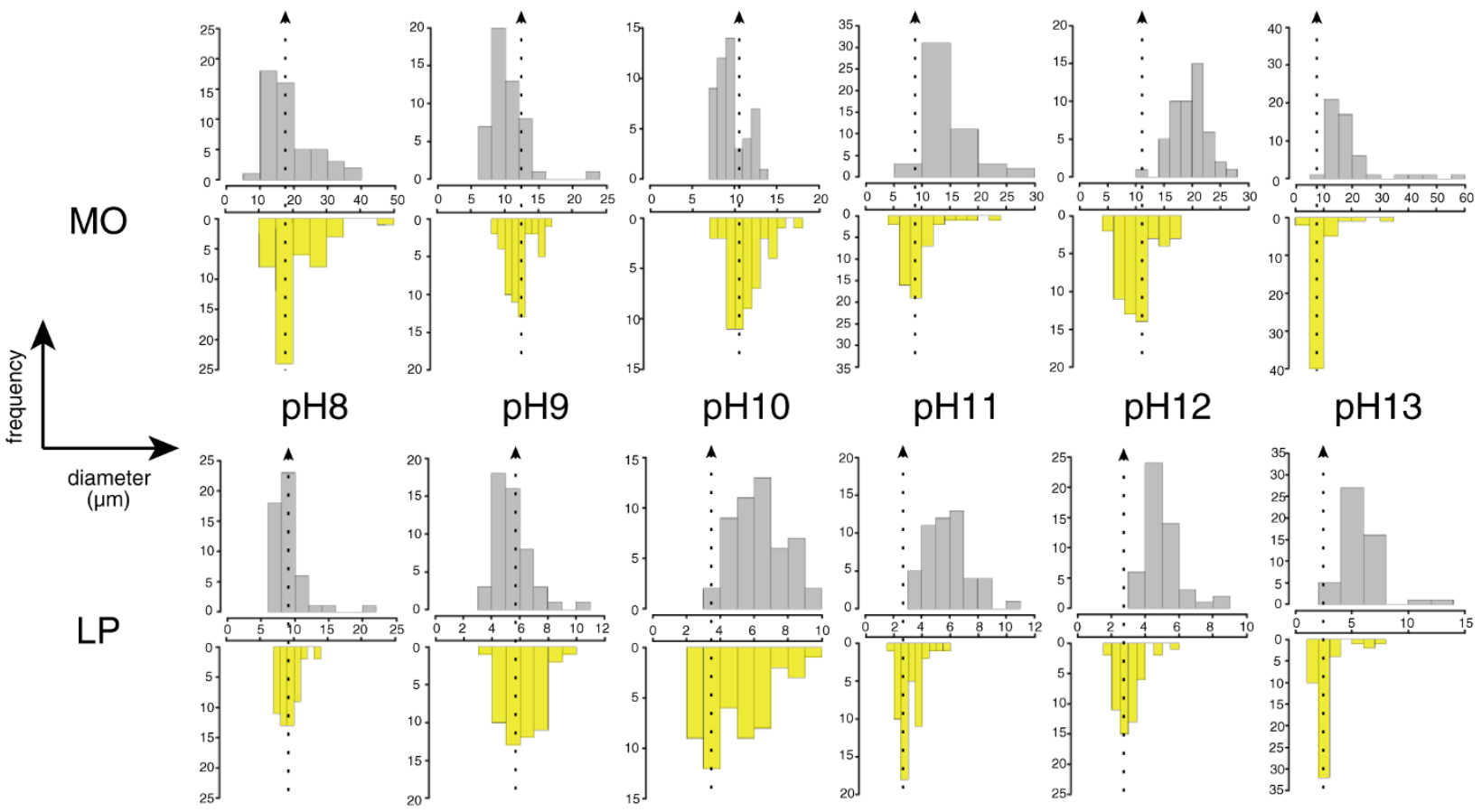

b

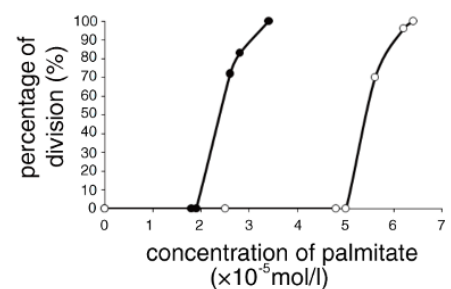

C

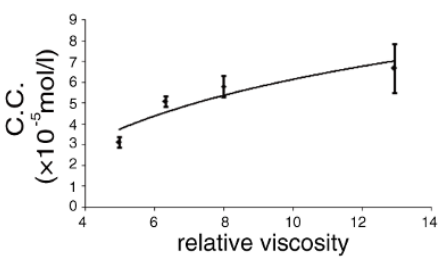

d

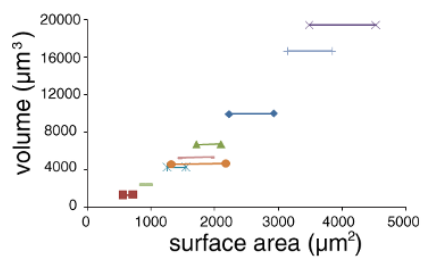

e

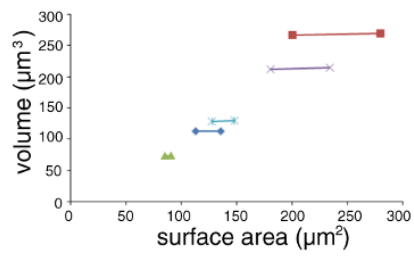

Figure $3 \mid$ The surface area increase induced by the incorporation of palmitate generated from the hydrolysis of pNPP. (a). Histograms showing distributions of the droplet cell diameters at $\mathrm{t}=0$ (gray bars) and $\mathrm{t}=5$ days (yellow bars) following the incubation at the indicated $\mathrm{pH}$ in mineral oil and liquid paraffin. $\mathrm{n}=50$, MO: mineral oil, LP: liquid paraffin. The most abundant diameter sizes following the droplet cell divisions are indicated by a dotted line with an arrowhead. (b). Percentages of divided droplet cells at the indicated concentrations of palmitate in mineral oil $(\bigcirc)$ and liquid paraffin (-). (c). The critical threshold concentrations (C.C.) of palmitate that triggers the droplet divisions in the solvent of the indicated relative viscosities. C.C. is the concentration of palmitate at which the division is initiated. The method of palmitate concentration is a described in Methods section. Average \pm s.d.; $\mathrm{n}=3$. One-way ANOVA test; $\mathrm{p}<0.01$. (d). The surface areas and volumes of individual droplet cells before and at the divisions in mineral oil. $\mathrm{n}=9$. (e). The surface areas and volumes of individual droplet cells (each shown by lines of distinct colors) before and at the divisions in liquid paraffin. $\mathrm{n}=5$.

represent the size of each droplet. The histogram was generated using statistics software package $\mathrm{R}$ (version 3.01).

Measurement of the droplet volume and surface area by laser confocal microscopy. The three-dimensional (3-D) reconstitution of individual amphipathic droplets was accomplished using ImageJ. The volume and surface area of each droplet was calculated from this 3-D image using Sync Measure 3D function of ImageJ.

Measurements of oil viscosity. The viscosities of various oils were determined using Ostwald viscometer, with water flow time at about $20.2 \mathrm{sec}$. The temperature was held constant at $25^{\circ} \mathrm{C}$ or $30^{\circ} \mathrm{C}$ in water bath, and the viscosity measurements were performed for three times for each sample and the average values were used for the viscosity calculations as follows:

$$
\begin{gathered}
\eta_{\mathrm{r}}=\eta / \eta_{\mathrm{w}}\left(\eta_{\mathrm{r}} \text { : relative viscosity, } \eta\right. \text { : coefficient of oil viscosity, } \\
\eta_{\mathrm{w}} \text { : coefficient of water viscosity) }
\end{gathered}
$$

Statistics. Statistical analyses were performed using the one-way ANOVA test.

1. Gonczy, P. Mechanisms of asymmetric cell division: flies and worms pave the way. Nature reviews. Molecular cell biology 9, 355-366 (2008).

2. Inaba, M. \& Yamashita, Y. M. Asymmetric stem cell division: precision for robustness. Cell stem cell 11, 461-469 (2012).
3. Andes-Koback, M. \& Keating, C. D. Complete Budding and Asymmetric Division of Primitive Model Cells To Produce Daughter Vesicles with Different Interior and Membrane Compositions. J Am Chem Soc 133, 9545-9555 (2011).

4. Bachmann, P. A., Walde, P., Luisi, P. L. \& Lang, J. Self-Replicating Reverse Micelles and Chemical Autopoiesis. J Am Chem Soc 112, 8200-8201 (1990).

5. Baumgart, T., Hess, S. T. \& Webb, W. W. Imaging coexisting fluid domains in biomembrane models coupling curvature and line tension. Nature 425, 821-824 (2003).

6. Browne, K. P., Walker, D. A., Bishop, K. J. M. \& Grzybowski, B. A. Self-Division of Macroscopic Droplets: Partitioning of Nanosized Cargo into Nanoscale Micelles. Angew Chem Int Edit 49, 6756-6759 (2010).

7. Kas, J. \& Sackmann, E. Shape transitions and shape stability of giant phospholipid vesicles in pure water induced by area-to-volume changes. Biophysical journal 60, 825-844 (1991).

8. Kurihara, K. et al. Self-reproduction of supramolecular giant vesicles combined with the amplification of encapsulated DNA. Nat Chem 3, 775-781 (2011).

9. Takakura, K. \& Sugawara, T. Membrane dynamics of a myelin-like giant multilamellar vesicle applicable to a self-reproducing system. Langmuir: the ACS journal of surfaces and colloids 20, 3832-3834 (2004).

10. Takakura, K., Toyota, T. \& Sugawara, T. A novel system of self-reproducing giant vesicles. J Am Chem Soc 125, 8134-8140 (2003).

11. Toyota, T. et al. Population study of sizes and components of self-reproducing giant multilamellar vesicles. Langmuir: the ACS journal of surfaces and colloids $\mathbf{2 4}$, 3037-3044 (2008). 
12. Wick, R., Walde, P. \& Luisi, P. L. Light-Microscopic Investigations of the Autocatalytic Self-Reproduction of Giant Vesicles. J Am Chem Soc 117, 1435-1436 (1995).

13. Yanagisawa, M., Imai, M. \& Taniguchi, T. Shape deformation of ternary vesicles coupled with phase separation. Physical Review Letters 100, 148102 (2008).

14. Walde, P., Han, D. \& Luisi, P. L. Spectroscopic and kinetic studies of lipases solubilized in reverse micelles. Biochemistry 32, 4029-4034 (1993).

15. Minc, N. \& Piel, M. Predicting division plane position and orientation. Trends in cell biology 22, 193-200 (2012).

16. Mercier, R., Kawai, Y. \& Errington, J. Excess membrane synthesis drives a primitive mode of cell proliferation. Cell 152, 997-1007 (2013).

\section{Acknowledgments}

We would like to acknowledge Drs. Satoshi Tomita for their numerous advices throughout the course of this work. This work was supported by Takeda Science Foundation (T.N.S.), Grant-in-aid for Young Scientists A (No. 24681028) (K.Y.) and a Grant-in-Aid for Challenging Exploratory Research (No. 23655156) (J.K.) from the Japan Society for the Promotion of Science (JSPS)

\section{Author contributions}

Y.S. performed all experiments. Y.S., K.Y., J.K. and T.N.S. all contributed to conceiving ideas and designing experiments. Y.S., K.Y., J.K. and T.N.S. wrote the manuscript.

\section{Additional information}

Supplementary information accompanies this paper at http://www.nature.com/ scientificreports

Competing financial interests: The authors declare no competing financial interests.

How to cite this article: Sato, Y., Yasuhara, K., Kikuchi, J.-I. \& Sato, T.N. Synthetic cell division system: Controlling equal vs. unequal divisions by design. Sci. Rep. 3, 3475; DOI:10.1038/srep03475 (2013)

(1) This work is licensed under a Creative Commons Attribution-

NonCommercial-NoDerivs 3.0 Unported license. To view a copy of this license, visit http://creativecommons.org/licenses/by-nc-nd/3.0 